\section{Appointment-Based Models and Medication Synchronization: Silver Bullet for Adherence or One Piece of the Puzzle?}

In this issue of JMCP, Holdford and Saxena ("Impact of Appointment-Based Medication Synchronization on Existing Users of Chronic Medcations") report on a claims analysis comparing the adherence rates (proportion of days covered [PDC]) of patrons electing to have their medications synchronized with a sample of patrons not participating in the program, matched within a decile based on past adherence rates. This article, and its predecessor, ${ }^{1}$ are meaningful first steps and logical uses of secondary prescription claims data for analyzing the role of medication synchronization programs in health care.

For persons choosing to have their medications synchronized to an appointment date, this study provides evidence that medication synchronization meets a specific, relevant need and facilitates medication adherence for enrollees as a whole. While beyond the scope of the aforementioned studies, there remains a need to understand patients that do not sign up or those that drop out and how pharmacy can best serve these individuals. Additional research is especially important given the complex nature of medication adherence and recent incentives for community pharmacies to help increase adherence rates for their patrons. ${ }^{2}$

The synchronization of refills as part of appointment-based refill programs addresses an important fraction of the reasons why patients may not take their prescriptions as directed. Research suggests that another important reason for nonadherence is patients' beliefs about medicines. ${ }^{3}$ Patients may have concerns about side effects, doubts about the importance of the medication, feelings that they have too many medications, or perceptions that their bodies are particularly sensitive to medications and require a smaller dose. ${ }^{4}$ These beliefs can result in patients making reasoned decisions about following or modifying their prescribers' suggestions for using the medications. This may include splitting tablets, taking partial doses, skipping doses, and discontinuing medications-activities that could put a serious wrench in the synchronization schedule, not to mention the effectiveness of the treatment in controlling their conditions.

Cost is another important factor and has been reported in the literature to be a barrier to adherence. ${ }^{5}$ Having all medication copays come due at once may not fit into some patients' budgets. Patients also may be using multiple pharmacies, which could make synchronization only partially address overall adherence. ${ }^{6}$ Many patients with multiple chronic conditions have complex regimens that are continually being adjusted. In these scenarios, the logistics of long-term synchronization would be challenging.

Promisingly, appointment-based refill programs call for a regular dialogue between the patient and the pharmacist where all of the aforementioned issues hypothetically could be addressed. It is not known, however, if these interactions give patients the opportunity to express their medication beliefs and air their concerns. Pharmacies are busy, and fitting in patient-centered discussions about adherence can be challenging in current workflows. ${ }^{7}$ Another concern is that the efficiencies gained through medication synchronization would not be reallocated into increased patient interactions, but rather would go to prescription-dispensing tasks, similar to what has been seen with increasing use of robotics. ${ }^{8}$ Even if the program can divert more time to patient interactions, there is evidence that some pharmacists may prioritize investigating the technical reasons for nonadherence, such as the patient having an extra supply due to a recent hospitalization or a changed dose where a new prescription was not written, over inquiring about medication beliefs. ${ }^{9-11}$
While the ideal for appointment-based models and medication synchronization programs is attractive, patients with tendencies toward the aforementioned belief, cost, or other reasons for nonadherence may be hesitant to enter into such a scheduling agreement with their pharmacies. This can leave a lot of patients out who could potentially benefit from an adherence intervention but want to avoid embarrassment and challenges to their autonomy by a pharmacist. There remains a need to understand how pharmacists can best address nonadherence during routine care.

Additional next steps include investigating how services such appointment-based refills are addressing the range of reasons why patients are nonadherent in addition to the objective of medication supply organization achieved through refill synchronization and reminder phone calls. Also, little is known about the patient experience with the service and what patients actually are doing at home. After all, a PDC $>80 \%$ is only a surrogate marker for a medication regimen in which the patient is invested to achieve health goals. And, while likely not the norm, for all we know patients are hoarding a cache of pills that have been picked up on their sync date with no intention of taking as their physician has prescribed.

\section{Matthew Witry, PharmD, PhD \\ University of Iowa College of Pharmacy; matthew-witry@uiowa.edu}

\section{DISCLOSURES}

The author declares no conflicts of interest.

\section{REFERENCES}

1. Holdford DA, Inocencio TJ. Adherence and persistence associated with an appointment-based medication synchronization program. J Am Pharm Assoc. 2013;53(6):576-83.

2. Owen JA. Medicare star ratings: stakeholder proceedings on community pharmacy and managed care partnerships in quality. J Am Pharm Assoc. 2014;54(3):228-40

3. Horne R. Patients' beliefs about prescribed medicines and their role in adherence to treatment in chronic physical illness. J Psychosom Res. 1999;47(6):555-67.

4. Britten N. Patients ideas about medicines: a qualitative study in a generalpractice population. Br J Gen Pract. 1994;44(387):465-68.

5. Piette JD, Heisler M, Wagner TH. Cost-related medication underuse among chronically ill adults: the treatments people forgo, how often, and who is at risk. Am J Pub Health. 2004;94(10):1782-87.

6. Look KA, Mott DA. Multiple pharmacy use and types of pharmacies used to obtain prescriptions. J Am Pharm Assoc. 2012;53(6):601-10.

7. Witry MJ, Doucette WR, Zhang Y, Farris KB. Multiple adherence tool evaluation study (MATES). J Manag Care Spec Pharm. 2014;20(7):734-40. Available at: http://www.amcp.org/WorkArea/DownloadAsset. aspx?id=18262.

8. Cavaco AM, Krookas AA. Community pharmacies automation: any impact on counselling duration and job satisfaction? Int J Clin Pharm. 2014;36(2):325-35.

9. Witry MJ, Doucette WR. Community pharmacists, medication monitoring, and the routine nature of refills: a qualitative study. J Am Pharm Assoc. 2014;54(6):594-603.

10. de Oliveira DR, Shoemaker SJ. Achieving patient centeredness in pharmacy practice: openness and the pharmacist's natural attitude. J Am Pharm Assoc. 2006;46(1):56-66.

11. Koster ES, van Meeteren MM, van Dijk M, et al. Patient-provider interaction during medication encounters: a study in outpatient pharmacies in the Netherlands. Patient Educ Couns. 2015;98(7):843-48. 